\title{
Indicadores de comportamiento de la competencia de innovación en el ámbito académico y en el profesional: revisión de la literatura
}

Aznar-Mas, Lourdes Emilia ${ }^{a}$, Pérez-Peñalver, María Joséb, Montero-Fleta, Begoña ${ }^{c}$, González-Ladrón de Guevara, Fernando Raimundo ${ }^{\text {, }}$ Marin-Garcia, Juan Antonio ${ }^{\mathrm{e}}$, Atarés-Huerta, Lorena María

${ }^{a}$ EICE IEMA, Dpto. Lingüística Aplicada, UPV, laznar@idm.upv.es, ${ }^{b}$ EICE IEMA, Dpto. Matemática Aplicada, UPV, mjperez@mat.upv.es, ${ }^{c} E I C E$ IEMA, Dpto. Lingüística Aplicada, UPV, bmontero@idm.upv.es, , ${ }^{\mathrm{d} E I C E}$ IEMA, Dpto. Organización de Empresas, UPV, fgonzal@omp.upv.es, ${ }^{e} E I C E$ IEMA, Dpto. Organización de Empresas, UPV jamarin@omp.upv.es , fEICE IEMA, Dpto. Tecnología de Alimentos, UPV, loathue@tal.upv.es

\footnotetext{
Abstract

Companies pay more and more attention to the innovation competence of their employees. Innovation is understood as either the introduction of something such as a product, service, process or method, completely new, or the improvement of something that already exists.
}

This paper presents the work performed by the research group IEMA-UPV so as to improve the INCODE barometer (from their previous European project "Innovation Competencies Development"), to be used by both companies and universities. This barometer has been adopted and will be refined in the framework of their new European project FINCODA ("Framework for Innovation Competencies Development and Assessment") as a tool to measure and assess the behavioral indicators of the innovation competence, which are being identified through a systematic literature review.

As a part of the research being performed, this paper also introduces the new model established by the FINCODA project. In this model, the innovation competence is presented as a cluster with three dimensions: creativity, critical thinking and the third one, intrapreneurship, which includes three sub-dimensions (initiative, teamwork and networking).

Keywords: Behavioral Indicators, Innovation Competence, Creativity, Critical Thinking, Intrapreneurship, Initiative, Teamwork, Networking.

\section{Resumen}

Las empresas valoran cada vez más la competencia de innovación de sus empleados, entendiendo por innovación la introducción de algo (producto, servicio, proceso o método) completamente nuevo o también la mejora de algo que ya existe.

Este trabajo presenta el trabajo realizado por el grupo de investigación IEMA-UPV para la mejora del barómetro INCODE (de su proyecto europeo 
Indicadores de comportamiento de la competencia de innovación en el ámbito académico y en el profesional: revisión de la literatura

anterior “Innovation Competencies Development”), y la utilización de este tanto en la empresa como en la universidad. Este barómetro ha sido adoptado y será mejorado en el marco de su nuevo proyecto europeo FINCODA ("Framework for Innovation Competencies Development and Assessment”) como instrumento para medir y evaluar los indicadores del comportamiento de la competencia de innovación, que están siendo identificados como resultado de una revisión sistemática de la literatura.

Como parte de la investigación que se está realizando, en este trabajo se presenta, asimismo, el nuevo modelo establecido por el proyecto FINCODA, en el que la competencia de innovación se muestra como un conjunto en el que se encuentran integradas tres dimensiones: creatividad, pensamiento crítico y una tercera, intraemprendedurismo, que incluye tres subdimensiones (iniciativa, trabajo en equipo y trabajo en red).

Palabras clave: indicadores de comportamiento, competencia de innovación, creatividad, pensamiento crítico, intraemprendedurismo, iniciativa, trabajo en equipo, trabajo en red.

\section{Introducción}

Las empresas y organizaciones se encuentran en un momento imparable de búsqueda y de superación de los retos del siglo XXI y han puesto énfasis en el potencial que proporciona la innovación a la hora de adaptarse a las nuevas dinámicas de un mundo cada vez más globalizado y en cambio permanente. La competencia de innovación que tiene un empleado, o la que podría tener, es una cualidad que a cualquier equipo de recursos humanos le gustaría poder requerir en el momento de la selección de personal o de contratación. Además, la innovación es uno de los elementos que fomenta una mayor productividad y crecimiento de las empresas, y hace que estas puedan convertirse en referentes de impacto en sus respectivas áreas (Aznar-Mas et al., 2015).

La investigación académica realizada sugiere que las características y los comportamientos de los individuos en el lugar de trabajo son la base para la innovación en las empresas (Patterson et al., 2009). Sin embargo, Cormican y O'Sullivan (2004), Adams et al. (2006) y Patterson et al. (2009) coinciden también en la falta del marco apropiado que debe utilizarse en la selección, contratación y en la gestión de las personas innovadoras. A este respecto, Hammond et al. (2001) subrayan el vacío existente en la literatura que se ha publicado sobre la evaluación cuantitativa de cuáles son los predictores de la innovación a nivel individual.

El proyecto europeo INCODE (Innovation Competencies Development), llevado a cabo de 2011 a 2013, tuvo como objetivo garantizar el éxito de la transferencia de las ideas innovadoras al producto innovador, y de los servicios que dependían de la integración del conocimiento pedagógico a las actividades de innovación del entorno laboral. INCODE desarrolló un barómetro para su utilización como herramienta de evaluación de la innovación en el entorno universitario. El trabajo que ahora se presenta, parte del anterior

(c)) BY-NC-ND 2016, Universitat Politècnica de València 
proyecto europeo INCODE y se está llevando a cabo en el contexto del proyecto europeo FINCODA (Framework for Innovation Competencies Development and Assessment), en el que están implicadas las cinco universidades que forman el grupo de alianza estratégica CARPE (Consortium of Applied Research and Professional Education) junto con nueve empresas participantes.

El fin de esta investigación es el de ahondar, de forma exhaustiva, en el aspecto de los rasgos o características de la competencia de innovación en los empleados de las empresas, así como en el desarrollo del liderazgo y de la innovación. Para llevar a cabo dichos objetivos se establecen, en primer lugar, las definiciones de los conceptos fundamentales sobre los que se sustenta la investigación.

\subsection{Competencia}

Según Villa Sánchez y Poblete Ruiz (2007), la competencia se refleja en una actuación correcta en contextos diversos y situaciones académicas auténticas que están basadas en la activación del conocimiento, las normas, las técnicas, los procedimientos, las habilidades y destrezas, las actitudes y los valores. El Marco Europeo de Cualificaciones para el Aprendizaje Permanente (2008) añade a todo lo anterior los términos de responsabilidad y el de autonomía y en él se describe la competencia como la habilidad en la utilización del conocimiento, de las destrezas y habilidades personales y/o sociales, tanto en situaciones laborales como en situaciones académicas, en las áreas de desarrollo personal y de desarrollo profesional.

La competencia es ese "saber hacer" complejo que es el resultado de la integración y de la adaptación de las capacidades y destrezas a las situaciones que comparten características similares (Lasnier, 2000; Fernández March, 2010), o ese "saber hacer" complejo respecto a cómo actuar cuando se movilizan y se combinan de forma efectiva toda la variedad de recursos internos y externos en una serie de situaciones. Tardif (2006) establece que la competencia es combinatoria, está en proceso, es contextual, es evolutiva. En ese "saber hacer" complejo contextualizado podemos establecer tres categorías de complejidad que son las competencias, las capacidades y las destrezas.

La competencia está formada por una serie de capacidades y estas, a su vez, lo están por un conjunto de destrezas o habilidades que son las que se requieren para una actuación profesional más compleja. La competencia supone la organización de múltiples recursos de los que esta se sirve y que utiliza, y es necesario que, en el caso de los programas educativos, los tipos de resultados de aprendizaje se limiten. En cuanto a su desarrollo, es necesario un tiempo suficiente a lo largo del periodo de aprendizaje o de una carrera profesional. Son las situaciones las que favorecen la necesidad de elegir y combinar los recursos dando lugar a un aprendizaje consciente y más reflexivo. Al trabajar en situaciones que son cada vez más complejas, surge la movilización y la combinación de los recursos y esta ofrece mejores resultados. La capacidad es un "saber hacer" de dificultad media que integra las destrezas que requieren un conocimiento de los procedimientos y las 
condiciones. La destreza es el "saber hacer" más sencillo (Lasnier, 2000; Bessant et al., 2001; Fernández March, 2010).

\subsection{Innovación y comportamiento innovador en el trabajo}

Al hacer referencia a la innovación siempre se hace referencia al cambio (Tidd, 2000) y este cambio necesariamente aumenta su valor. La innovación se define como una introducción intencionada y su aplicación en un rol, dentro de un grupo o una organización de ideas, procesos, productos o procedimientos (West y Farr, 1989). La OCDE (OECD, 2005) define la innovación como la implementación de un producto (bienes o servicios) o proceso, un nuevo método de marketing, un nuevo método de organización en la práctica empresarial, entorno laboral o de relaciones externas que sea nuevo o que se haya mejorado de forma significativa. Para Rogers (2003) y Lehto et al. (2011) esta es la definición más sencilla de innovación y al mismo tiempo la mejor para el momento actual. Relaciona la innovación con la invención de algo nuevo o la mejora de algo que ya existe (o como una idea, práctica u objeto que se considera nuevo). La innovación está íntimamente ligada a la creatividad, "el momento de la creatividad de un proceso se refiere a la generación de ideas, y la innovación se refiere al siguiente estadio en el que se implementan ideas para conseguir mejores procedimientos, prácticas o productos" (Anderson, 2014, p. 4). Por consiguiente, se asume que la innovación es un proceso multinivel, con diferentes actividades y diferentes comportamientos individuales que son necesarios en cada uno de esos niveles.

Recientemente, ha surgido el concepto de comportamiento innovador en el trabajo (Innovative Work Behaviour "IWB") para referirse a los empleados que se implican en actividades innovadoras (De Spiegelaere et al., 2012). El comportamiento innovador en el trabajo aglutina una serie de actividades dirigidas hacia el reconocimiento, desarrollo, modificación, adopción e implementación de ideas (Van de Ven, 1986; Scott y Bruce, 1994). Es el reconocimiento de problemas junto con la iniciación e introducción intencionada de ideas nuevas y útiles, así como una serie de comportamientos que se necesitan para desarrollar, lanzar e implementar ideas con el propósito de fomentar la actuación personal o de la empresa (Farr y Ford, 1990; De Jong y Den Hartog, 2007).

\subsection{Evaluación del comportamiento e indicadores}

La evaluación del comportamiento se realiza a través de los aspectos que son claramente observables sobre la manera en la que un individuo interactúa con su entorno (GrothMarnat, 2003). Estos rasgos observables son los indicadores de comportamiento, que muestran la presencia de una competencia particular (Dent y Krefft, 2004) o la evidencia del grado de desarrollo de una competencia (Cruz Serna et al., 2012). Los descriptores que se busquen han de ser muestras de comportamiento observables y medibles para que permitan una mejor evaluación de la actuación del personal y, de este modo, de su gestión y de su desarrollo (Muchinsky, 2006). Cuantos más indicadores de comportamiento aparezcan durante una entrevista de trabajo, por ejemplo, mayor probabilidad habrá de que el candidato destaque en una determinada competencia (Dent y Krefft, 2004).

(cc) EY-NC-ND 2016, Universitat Politècnica de València 
La evaluación del comportamiento se centra en aspectos como, por ejemplo, el porqué de los comportamientos deseados o comportamientos meta, cómo se deberían medir, qué niveles de análisis, de posibilidad de cambio o de complejidad son los que se prefieren. Estas consideraciones son obvias respecto al uso de los procedimientos de evaluación específicos que se han diseñado para obtener datos de las medidas validadas y bien definidas de dichos comportamientos meta, y de las variables según cada contexto para un cliente individual (O’Brien et al., 2010). Además, la evaluación del comportamiento también fomenta el diseño de instrumentos de medida que sean susceptibles de ser validados y estandarizados, reduciendo así la subjetividad de los procesos de evaluación de personal (Arias y Heredia, 2006).

\section{Objetivos}

Los objetivos de este trabajo son identificar y clasificar los indicadores de comportamiento de las personas innovadoras con el fin de proporcionar a las empresas una herramienta que sea eficaz para evaluarlos. Dicha herramienta debe poder observar y medir el comportamiento de los empleados respecto a la competencia de innovación. Este trabajo surge de la investigación que se está realizando en FINCODA, a través de una revisión sistemática de la literatura, para diseñar un modelo de la competencia de innovación con las dimensiones y comportamientos de cada dimensión, que serán evaluadas con el barómetro INCODE una vez mejorado y actualizado para los nuevos indicadores.

\section{Metodología}

Para llegar al Modelo FINCODA de Competencia de Innovación, el grupo de investigación IEMA (Innovación en la Evaluación para la Mejora del Aprendizaje Activo) de la Universitat Politècnica de València ha seguido tres pasos. Es necesario comentar, previamente, que este modelo no está basado en datos estadísticos sino en la revisión sistemática de la literatura que se ha realizado con empresas e investigadores, tal y como se describe a continuación.

El primer paso ha sido la utilización de bases de datos como Scopus, Web of Knowledge o la biblioteca digital de IEEE Xplore para la identificación de las dimensiones que la literatura consideraba relevantes en lo que concierne a comportamiento innovador, todo ello apoyado en trabajos de autores durante el periodo estudiado, de 2000 a 2015. Las dimensiones resultantes de la investigación no proceden exactamente de los nombres dados por los autores en la literatura sino que han sido ancladas sobre las definiciones de los constructos o dimensiones del modelo. La revisión ha sido llevada a cabo por tres equipos de investigadores del grupo de investigación IEMA, de la UPV (Marin-Garcia et al., 2015; Montero-Fleta et al., 2016; Pérez-Peñalver et al., 2016) que han estudiado en profundidad alrededor de una treintena de artículos de entre aproximadamente 1000 referencias entre las más relevantes.

(cc) EY-NC-ND 2016, Universitat Politècnica de València

Congreso In-Red (2016) 
El segundo paso ha sido la evaluación de las dimensiones y sus correspondientes definiciones por tres representantes de las empresas multinacionales españolas que participan en el proyecto junto con cuatro investigadores, para detectar cualquier posible omisión, detalle superfluo o inconsistencia en el modelo de competencia de innovación propuesto y en las etiquetas usadas para definir cada dimensión o constructo.

El objetivo del tercer paso ha sido reutilizar una lista de indicadores de comportamiento que se había usado ya en estudios previos relacionados con la competencia de innovación. Estos indicadores se han asociado posteriormente con cada dimensión del modelo, basándose en la clasificación cruzada realizada por los autores de la investigación de la literatura y del criterio del grupo de investigación IEMA, de la UPV. Debe destacarse que el modelo resultante de competencia de innovación es más rico que las dimensiones originales propuestas por los autores, que no coinciden con las del modelo que se presenta en este trabajo.

Con el fin de mejorar y validar el modelo logrado a través de las tres fases descritas previamente, se han llevado a cabo una serie de actividades antes y durante las reuniones generales de miembros del proyecto FINCODA que han tenido lugar en Valencia en enero de 2016 y en Utrecht en mayo de 2016. Cabe destacar que las aportaciones de las empresas participantes presentes en las reuniones han sido de vital importancia. Como resultado de esos encuentros se ha establecido finalmente un prototipo que ya se encuentra en fase de validación en este momento por parte de las universidades y de las empresas miembros de FINCODA.

\section{Resultados}

El Modelo FINCODA de Competencia de Innovación que se presenta (figura 1) está basado en tres dimensiones o constructos: Creatividad, Pensamiento Crítico e Intraemprendedurismo. Este último es un conjunto de tres sub-dimensiones que incluye la Iniciativa, Trabajo en equipo y Trabajo en red. La evaluación de competencias es una tarea compleja que incluye decidir sobre varios aspectos o dimensiones de la competencia (Marin-Garcia et al., 2015). Este prototipo es el resultado del refinamiento inicial del barómetro desarrollado para el proyecto INCODE para su adaptación al presente proyecto FINCODA (Framework for Innovation Competence Development and Assessment), que se ha descrito en la sección previa de este trabajo. 


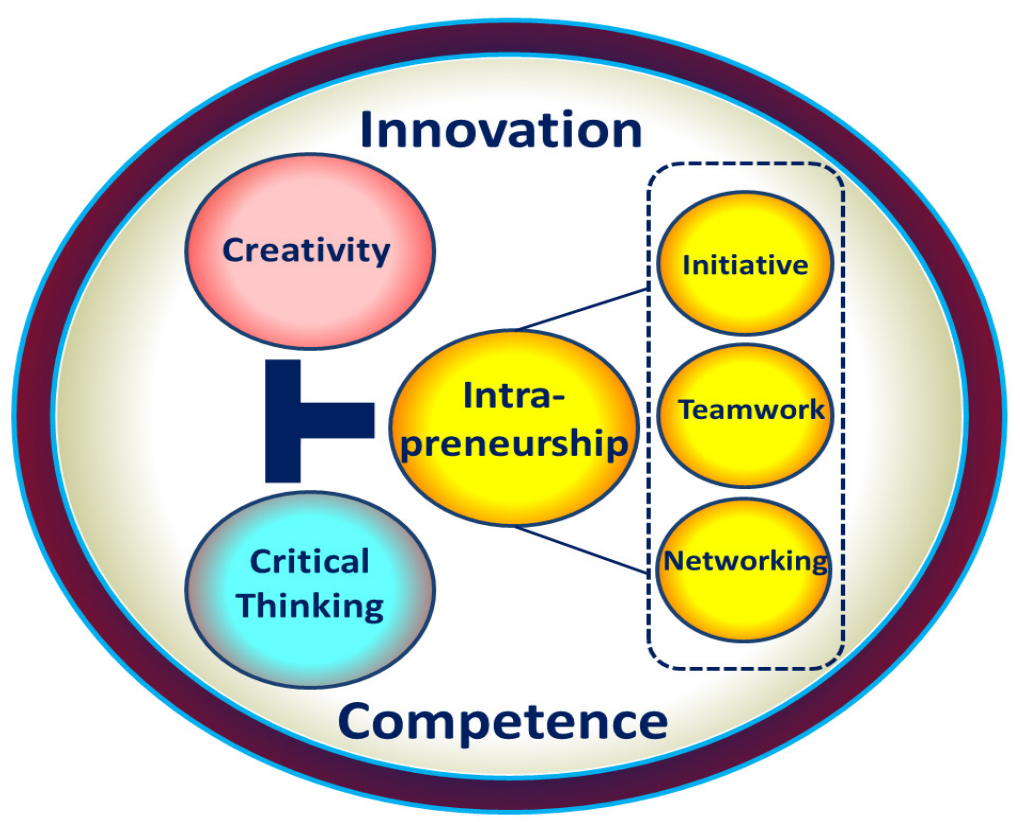

Figura 1. (C) FINCODA UPV-SEE-CSP team (2016). Innovation Competence Model

La investigación en curso está centrada en la descripción definitiva y la validación de cada dimensión y de los indicadores de comportamiento correspondientes a cada dimensión o constructo de la Competencia de Innovación, que son los que van a dar forma al barómetro FINCODA. El resultado final será un instrumento que se utilice para evaluar o para contribuir a la evaluación del desarrollo de la competencia de innovación de los empleados o de los candidatos para un puesto de trabajo, y que pueda adaptarse a las necesidades de las empresas.

En la tabla 1 se encuentran algunos ejemplos de indicadores de comportamiento innovador que se han identificado tanto en la revisión de la literatura como a través del trabajo y actividades desarrollados por los investigadores. Los indicadores se muestran agrupados y clasificados según la dimensión a la que pertenecen, de acuerdo con el Modelo FINCODA de competencia de innovación.

Tabla 1. Ejemplos de indicadores de comportamiento innovador

\begin{tabular}{|l|l|}
\hline CREATIVITY \\
\hline Present novel ideas & (Watts et al., 2012; Scott \& Bruce, 1994) \\
\hline $\begin{array}{l}\text { Generate original solutions for problems or } \\
\text { to opportunities }\end{array}$ & $\begin{array}{l}\text { (De Spiegelaere et al., 2012; Janssen, } \\
2000)\end{array}$ \\
\hline CRITICAL THINKING & \\
\hline $\begin{array}{l}\text { Evaluate the advantages and disadvantages } \\
\text { of actions or ideas }\end{array}$ & (Watts et al., 2012; Kleysen \& Street, \\
\hline $\begin{array}{l}\text { Develop and experiment with new ways of } \\
\text { problem solving }\end{array}$ & (Choi, 2004) \\
\hline
\end{tabular}

\section{(cc) EY-NC-ND 2016, Universitat Politècnica de València}

Congreso In-Red (2016) 


\begin{tabular}{|l|l|}
\hline INITIATIVE \\
\hline $\begin{array}{l}\text { Convince people to support an innovative } \\
\text { idea }\end{array}$ & $\begin{array}{l}\text { (De Spiegelaere et al., 2012; Kleysen \& } \\
\text { Street, 2001) }\end{array}$ \\
\hline $\begin{array}{l}\text { Use an action-based approach: develop } \\
\text { goals, collect information, make plans for } \\
\text { executing them }\end{array}$ & $\begin{array}{l}\text { (Patterson et al., 2009; De Spiegelaere et } \\
\text { al., 2012) }\end{array}$ \\
\hline TEAMWORK \\
\hline $\begin{array}{l}\text { Interact effectively with others to facilitate } \\
\text { the gathering, integrating and conveying of } \\
\text { information in many forms }\end{array}$ & (Watts et al., 2012; Choi, 2004) \\
\hline $\begin{array}{l}\text { Identify sources of conflict between oneself } \\
\text { and others, or among other people, and to } \\
\text { take steps to overcome disharmony }\end{array}$ & (Berdrow \& Evers, 2010; Watts et al., \\
\hline NETWORKING \\
\hline $\begin{array}{l}\text { Identify the appropriate decision makers } \\
\text { and stakeholders who have the power to } \\
\text { make a difference }\end{array}$ & (Watts et al., 2012) \\
\hline $\begin{array}{l}\text { Build relationships outside the } \\
\text { team/organization }\end{array}$ & (Ferris et al., 2005) \\
\hline
\end{tabular}

\section{Conclusiones}

El proyecto europeo INCODE presentó como resultado un barómetro como herramienta de evaluación de la innovación en el contexto universitario. El presente trabajo, desarrollado en el marco del proyecto europeo FINCODA y que tiene su origen en el proyecto INCODE, pretende identificar y clasificar los indicadores de comportamiento de las personas innovadoras para proporcionar a las empresas una herramienta útil que permita observar y medir el comportamiento de los empleados en relación a la competencia de innovación. A tal fin, y en primer lugar, se ha desarrollado una revisión sistemática de la literatura con el objetivo de diseñar un modelo que incluya las competencias básicas de innovación y una serie de comportamientos deseados que serán evaluados con el barómetro INCODE, una vez mejorado y actualizado para los nuevos indicadores.

El modelo FINCODA de la competencia de innovación que se está validando en la actualidad está basado en tres dimensiones: Creatividad, Pensamiento Crítico e Intraemprendedurismo. En el caso del Intraemprendedurismo se incluyen tres subdimensiones: Iniciativa, Trabajo en equipo y Trabajo en red.

El grupo de universidades participantes está formado por: Hamburg University of Applied Sciences (Hochschule für Angewandte Wissenschaften Hamburg, Alemania), Manchester Metropolitan University (MMU, Reino Unido), Turku University of Applied Sciences (Turun Ammattikorkeakoulu TUAS, Finlandia), Universitat Politècnica de València (UPV, España) y University of Applied Sciences Utrecht (HU, Holanda).

Las empresas participantes son: Elomatic Ltd. (Finlandia), Meyer Turku Oy (Finlandia), Lactoprot (Alemania), ECDL (Holanda), John Caunt Scientific Ltd. (Reino Unido), Carter\&Corson Partnership Ltd. (Reino Unido), Celestica (España), Schneider Electric España S.A. (España) y Enterprise Europe Network North West EENNW (Reino Unido).

(cc) EY-NC-ND 2016, Universitat Politècnica de València 


\section{Agradecimientos}

Este trabajo ha sido financiado con el Proyecto Erasmus+ Project FINCODA, Framework for Innovation Competences Development and Assessment_554493-EPP-1-2014-1-FIEPPKA2-KA (The European Commission support for the production of this publication does not constitute an endorsement of the contents which reflects the views only of the authors, and the Commission cannot be held responsible for any use which may be made of the information contained therein) y el Proyecto PIME 2015-2016 A/09_Evaluación de los indicadores del comportamiento innovador en el alumno universitario, de la Universitat Politècnica de València.

\section{Referencias}

ADAMS, R., BESSANT, J., PHELPS, R. (2006). "Innovation management measurement: A review". International Journal of Management Reviews, 8(1), 21-47.

ANDERSON, N., POTOČNIK, K., ZHOU, J. (2014). "Innovation and creativity in organizations a state-of-the-science review, prospective commentary, and guiding framework". Journal of Management, 40(5), 1297-1333.

ARIAS GALICIA L. F., HEREDIA ESPINOSA, V. (2006). Administración de Recursos Humanos para el Alto Desempeño (6th ed.). México: Trillas.

AZNAR-MAS, L. E., MONTERO FLETA, B., PÉREZ PEÑALVER, M. J. et al. (2015). “De Proyecto INCODE a FINCODA: utilización del Barómetro INCODE en alumnos universitarios y en empleados con competencias de innovación" en Actas Congreso InRed 2015. 30 junio-1 julio 2015, Universitat Politècnica de València, 1197-1206.

BERDROW, I., EVERS, F. T. (2010). "Bases of competence: an instrument for self and institutional assessment”. Assessment \& Evaluation in Higher Education, 35(4), 419-434.

BESSANT, J., CAFFYN, S., GALLAGHER, M. (2001). "An evolutionary model of continuous improvement behavior”. Technovation, 21(2), 67-77.

CARPE. Consortium on Applied Research and Professional Education. $<$ http://husite.nl/carpenetwork $>$ [Consulta: 7 de marzo de 2016]

CHOI, J. N. (2004). "Individual and contextual dynamics of innovation-use behavior in organizations". Human Performance, 17(4), 397-414.

CORMICAN, K., O'SULLIVAN, D. (2004). “Auditing best practice for effective product innovation management”. Technovation, 24(10), 819-829.

CRUZ SERNA, A. V., OROZCO JARAMILlO, Á. M., VARELA GAVIRIA, G., et al. (2012). Competencias gerenciales del directivo docente, para el ejercicio efectivo de la gerencia educativa. Identificación, descripción y metodologías para su desarrollo. (Trabajo de Grado). Facultad de Educación. Universidad de San Buenaventura, Medellín (Colombia).

(cc) BY-NC-ND 2016, Universitat Politècnica de València

Congreso In-Red (2016) 
Indicadores de comportamiento de la competencia de innovación en el ámbito académico y en el profesional: revisión de la literatura

DE JONG, J. P. J., DEN HARTOG, D. N. (2007). "How leaders influence employees' innovative behavior". European Journal of Innovation Management, 10(1), 41-64. $<$ http://doi.org/10.1108/14601060710720546> [Consulta: 8 de marzo de 2016]

DE SPIEGELAERE, S., VAN GYES, G., VAN THILLO, T. et al. (2012). "Innovative Work Behavior: Concept \& Measurement” en ISPIM Conference Proceedings, 1.

DENT, S. M., KREFFT, J. H. (2004). Powerhouse Partners: A Blueprint for Building Organizational Culture for Breakaway Results. California: Davies Black Publishing.

EUROPEAN PARLIAMENT COUNCIL. Recommendations (Recommendation of the European Parliament and of the Council of 23 April 2008 on the establishment of the European Qualifications Framework for lifelong learning). Official Journal C 111, 1-7. < http://eurlex.europa.eu/legal-content/EN/TXT/?uri=celex:32008H0506\%2801\%29>

FARR, J. L., FORD, C. M. (1990). "Individual innovation” en West, M. A., Farr, J. L. (1990), Innovation and creativity at Work: Psychological and Organisational Strategies, 63-80. Chichester: John Wiley.

FERNÁNDEZ MARCH, A. (2010). La evaluación orientada al aprendizaje en un modelo de formación por competencias en la educación universitaria. Revista de Docencia Universitaria, 8(1), 11-34.

FERRIS, G.R., TREADWAY, D.C., KOLODINSKY, R.W., et al. (2005). "Development and validation of the political skill inventory". Journal of Management, 31(1), 126-152.

FINCODA. Framework for Innovation Competences Development and Assessment. Erasmus ${ }^{+}$Project: 554493-EPP-1-2014-1-FI-EPPKA2-KA

GROTH-MARNAT, G. (2009). Handbook of psychological assessment. New York: John Wiley \& Sons.

HAMMOND, M. M., NEFF, N. L., FARR, J. L., et al. (2011). Predictors of individual-level innovation at work: A meta-analysis. Psychology of Aesthetics, Creativity, and the Arts, $5(1), 90-105$

JANSSEN, O. (2000). "Job demands, perceptions of effort-reward fairness and innovative work behavior”. Journal of Occupational and Organizational Psychology, 73, 287-302.

KLEYSEN, R. F., STREET, C. T. (2001). "Toward a multi-dimensional measure of individual innovative behavior". Journal of Intellectual Capital, 2(3), 284-296.

LASNIER, F. (2000). Réussir la formation par competences. Montreal: Guérin

INCODE. Innovation Competencies Development. Erasmus ${ }^{+}$Project: $<$http://www.incodeeu.eu/en> [Consulta: 8 de marzo de 2016]

(cc) EY-NC-ND 2016, Universitat Politècnica de València 
LEHTO, A., KAIRISTO-MERTANEN, L., PENTTILÄ, T. (eds.) (2011). Towards Innovation Pedagogy. A new approach to teaching and learning for universities of applied sciences. Reports from Turku University of Applied Sciences 100. Tampere: Tampereen Yliopistopaino-Juvenes Print Oy.

MARIN-GARCIA, J. A., RAMÍREZ BAYARRI, L., ANDREU-ANDRÉS, M. A. (2015). "Comparación de los métodos de escalas y frecuencia de comportamiento para valorar la competencia de innovación. El punto de vista de alumnos y profesor en el caso de una asignatura de máster" en Actas Congreso InRed 2015. 30 junio-1 julio 2015, Universitat Politècnica de València, 1113-1126.

MARIN-GARCIA, J. A., RAMÍREZ BAYARRI, L., ATARÉS-HUERTA, L. M. (2015). "Protocol: Comparing advantages and disadvantages of Rating Scales, Behavior Observation Scales and Paired Comparison Scales for behavior assessment of competencies in workers. A systematic literature review". WPOM-Working Papers on Operations Management, 6(2), 49-63.

MONTERO-FLETA, B., PÉREZ-PEÑALVER, M. J., AZNAR-MAS, L. E. (2016). "Behavioural indicators of innovators. A search protocol for a systematic literature review". Procedia Social and Behavioral Sciences (pendiente de publicación).

MUCHINSKY, P. M. (2006). Psychology applied to work: An introduction to industrial and organizational psychology, 1. North Carolina: Thomson Wadsworth.

O'BRIEN, W. H., OEMIG, C. K., NORTHERN, J. J. (2010). "Behavioral assessment with adults" en Handbook of clinical psychology competencies, 283-308. New York: Springer.

OECD/Eurostat (2005). Oslo Manual: Guidelines for Collecting and Interpreting Innovation Data (3rd ed.). The Measurement of Scientific and Technological Activities. Paris: OECD Publishing.

PATTERSON, F., KERRIN, M., GATTO-ROISSARD, G. (2009). Characteristics and behaviours of innovative people in organisations. Literature Review. NESTA Policy Research Unit. City University, London.

PÉREZ-PEÑALVER, M. J., WATTS, F., MARIN-GARCIA, J. A. et al. (2016). "Behavioral indicators of innovation competence" en Proceedings of INTED Conference 7th - 9th March 2016, Valencia (Spain), 8606-8611.

ROGERS, E. M. (2003). Diffusion of Innovations (5th ed.). New York: Free Press.

SCOTT, S. G., BRUCE, R. A. (1994). "Determinants of innovative behavior: A path model of individual innovation in the workplace". Academy of Management Journal, 37(3), 580-607.

TARDIF, J. (2006). L'évaluation des compétences. Documenter le parcours de développement. Montréal: Chenelière Éducation.

(cc) EY-NC-ND 2016, Universitat Politècnica de València

Congreso In-Red (2016) 
Indicadores de comportamiento de la competencia de innovación en el ámbito académico y en el profesional: revisión de la literatura

TIDD, J. (2000). The competence cycle: Translating knowledge into new processes, products and services. From Knowledge Management to Strategic Competence: Measuring Technological, Market and Organizational Innovation. London: Imperial College Press.

VAN DE VEN, A. H. (1986). "Central problems in the management of innovation". Management Science, 32, 590-607.

VILlA SÁNCHEZ, A., POBLETE RUIZ, M. P. (2007). Aprendizaje basado en competencias. Bilbao: Universidad de Deusto.

WATTS, F., MARIN-GARCIA, J. A., GARCÍA-CARBONELL, A., AZNAR-MAS, L. E. (2012). "Validation of a rubric to assess innovation competence" en Working Papers on Operations Management, 3(1), pp. 61-70.

WEST, M. A., FARR, J. L. (1989). "Innovation at work: Psychological perspectives". Social Behavior, 4, 15-30. 